\title{
Dr. Ajaya Bhattarai research works with the future proposed research
}

\author{
Contributor: , Ajaya Bhattarai ${ }^{1}$ \\ 1, Tribhuvan University; bkajaya@yahoo.com
}

Version received: 17 May 2020

check for

updates

\section{Abstract}

I am Dr. Ajaya Bhattarai, an Assistant Professor of Chemistry at Tribhuvan University, Nepal. I have received my M.Sc. degree in Physical Chemistry from Tribhuvan University in 1998 and a Ph.D. degree in Polymer Chemistry from North Bengal University, India in 2010. I have also received the "TWAS Young Scientist Award" in 2008 for Chemistry. I have visited the University of Warsaw, Poland for Post-doctorate research in Nanomaterials as well as Surface Chemistry in 2013 under Erasmus Mundus post-doctoral fellowship of European Commission for nine months. I was at Boston College, the USA for Post-doctorate research in "Theoretical studies of Glass-Forming liquids" under Fulbright fellowship for the year 2018-2019. I have published more than 100 articles in reputed journals. My current research interest is in Polymer Chemistry, Surface Chemistry, and Nanomaterials as well as theoretical studies of Glass-Forming liquids.

Table of Contents [Hide]

\section{Dr. Ajaya Bhattarai research works with the future proposed research}

My research in the past several years has focused on a systematic and comprehensive study of the solution behaviour of a polyelectrolyte in mixed solvent media using conductometry, densitometry, and viscometry. This is a very important problem because

1) Polyelectrolytes are the simplest way to boost the viscosity of aqueous coatings which are likely to play an even more significant role in the coatings industry as time goes on, owing to environmental concerns from the use of organic solvents.

2) Unlike neutral polymers which only have molar mass (chain length) and concentration influencing viscosity and viscoelastic character of their solutions, polyelectrolyte solutions are also influenced by the effective charge on the chain and salt concentration.

3) While other methods to determine the effective charge on the chain are available, conductivity is by far the simplest method and the only one that could ever be routinely used in industry for quality control.

My first publication of conductivity studies on polyelectrolyte in methanol-water mixed solvent media was able to provide me the "Third World Academy Sciences for Young Scientist (TWAS)" Award for the year 2008 in Chemistry (http://twas.ictp.it/publications/miscellaneous/annual-report-2008).

On that publication, there has been a great deal of work on interpreting data on the conductivity of polyelectrolyte solutions since Manning's work.

Our research group of Department of Chemistry, University of North Bengal, Darjeeling, India had been successful in developing the model for describing the electrical conductivity behaviour of polyelectrolyte solutions in the presence of an added salt 2,3 . Moreover, we had also been able to separate the intramolecular and intermolecular interactions in polyelectrolyte solutions which might help understand their characteristic solution behavior ${ }^{4,5}$. We have studied the solvodynamic properties of sodium polystyrene sulfonate in methanol-water mixed solvent media in the absence and 
the presence of salt using viscometric method ${ }^{6}$ and we also investigated the effect of different low-molar mass electrolytes on the viscometric behaviour of sodium polystyrene sulfonate in methanol-water mixed solvent media ${ }^{7}$.

In the year 2009, we found the Manning counterion condensation theory for salt-free polyelectrolyte solution failed to describe the experimental results. We analyzed the data based on a new model for semi-dilute polyelectrolyte conductivity which takes into account the scaling arguments to obtain the fractions of uncondensed counterions that were found to depend on the polyelectrolyte concentration. The effects of the temperature and the relative permittivity of the medium on the equivalent conductivity as well as on the fraction of uncondensed counterions were successfully discussed ${ }^{8}$.

Moreover, I independently measured the specific conductances of sodium polystyrene sulfonate in water and methanol-water mixture. The equivalent conductivity data against concentration were evaluated by the scaling concept. The effects of the solvent composition and the polyelectrolyte concentration on the fractions of uncondensed counterions, the polyion conductivities, the standard state free energies of counterion association, and the polyion transference number were calculated ${ }^{9}$. The charge density of sodium polystyrene sulfonate was used for my research article ${ }^{10}$ to see the variation of the critical aggregation concentration of cetyltrimethylammonium bromide in sodium polystyrene sulfonate against the charge density of sodium polystyrene sulfonate.

I also did huge work on the influence of the polyelectrolyte on the aggregation behaviour of an oppositely charged surfactant in mixed solvents using conductometry ${ }^{10}$, tensiometry ${ }^{11}$, and viscometry ${ }^{11}$.

After submission of my Ph.D. thesis from University North Bengal, Darjeeling, India, I started collaboration work with Professor Sujeet Kumar Chatterjee of my home Institute on the subject of Thermodynamics properties on molten alloys and we were able to publish the interesting article in the reputed journal on the Journal of Alloys and Compounds ${ }^{12}$.

I got 5 publications in the reputed journals in a very short time with Professor Chatterjee in the area of molten alloys and then I approached him to start new research laboratory for experimental works and we started the research work on surface chemistry with one Ph.D. student and then we were able to publish our first research article from surface chemistry on cationic surfactant (cetyltrimethylammounium bromide) in water and methanol-water mixed solvent media ${ }^{13}$ which was cited by the reputed journa $\mathrm{l}^{4}$ and has huge industrial viability in colloidal, emulsion technology, sol-gels and similar others. After seeing this publication, Professor Man Singh, New Delhi, India collaborated with our group and provided his invented equipment Man Singh Survismeter. Our two Ph.D. students and three Master students were used Mansingh Survismeter in their research work. One of my Ph.D. students Sujit Kumar Shah awarded Ph.D. and became Assistant Professor and then also received "Third World Academy Sciences for Young Scientist (TWAS)" Award for the year 2017 in Chemistry (https://twas.org/prizes-young-scientists-developing-countrieslist-winners) with the help of three reputed international publications ${ }^{5,16,17}$ and another my Ph.D. student Tulasi Prasad Niraula who was my teacher in my undergraduate studies who also received the Ph.D. award and now he is Associate Professor of Tribhuvan University in my guidance of international publications on anionic surfactant(Sodium dodecyl sulfate)with or without monovalent salts in water and methanol-water mixed solvent media ${ }^{18,19,20}$.

I not only shared my ideas with other chemists of the eastern part of Nepal but also guided them in the various projects $^{21}$. I have the opportunity to publish popular articles in Newspapers. Also, I have long experience in teaching and researches as well as I have reviewed several international Journals(https://publons.com/researcher/1579412/drajaya-bhattarai/).

I have extended the works of the interaction of cationic surfactant with anionic surfactant in watefe,23 and mixed solvent media $24,25,26$. As we know mixed surfactant systems are much favored from the view-point of economy and performance. They are less expensive than isomerically pure surfactants and they also often provide better performance. Surfactant-surfactant interactions have been extensive use in industrial, pharmaceutical, technological, and biochemical fields. In the pharmaceutical field, for example, mixed micelle has been found to enhance the absorption of various drugs in the human body. Several mixtures of cationic and anionic surfactant mixtures have been used in cleaning products to facilitate the dissolution and improved tolerance of water hardness. Due to their 
synergistic behavior at cmc, cosmetic industries use the mixed micelles in low concentrations to avoid potential skin irritation.

One of my Ph.D. students are working surfactants and dyes interaction in alcohol-water mixed solvent media. I have guided the Ph.D. students in the interaction of the cationic surfactant with anionic surfactant in the presence of dyes in the water at the Central University of Gujarat, India supported by TWAS, Italy ${ }^{27,28}$. In the same University, we synthesized metallo-surfactant complexes and worked on the hydrophobic study of increasing alkyl chain length of platinum surfactant complexes and did the characterization via thermogravimetric and surface morphology. Also, we studied the micellization and thermodynamics properties of surfactant and platinum surfactant complexes ${ }^{29}$.

I have also received Erasmus Mundus Scholarship through the EMEA scholarship program for Post-doctorate for the year 2012-2013 at the University of Warsaw, Poland. From that visit, I have made significant scientific contributions in experimental work on problems of transport and interactions of small molecules in microemulsions. Generally, I have looked for the pharmaceutics capsulation system. In detail, there are solutions for normal and inverted micelles. I have observed systems containing surfactant/ (calixarene, solvent, antioxidants and water) using UV-vis and DLS techniques with the help of Dr. Hanna Wilczura-Wachnik, Laboratory of the Physicochemical Fundamentals of Chemical Technology, University of Warsaw, Warsaw, Poland. From the experimental data, I have determined the thermodynamic parameters for the investigated system as, for example, a distribution constant between the solvent and micellar phase for small molecules, and others ${ }^{30,31,32}$. Moreover, I have studied strongly on novel techniques to produce one-dimensional and two-dimensional synthesized nanomaterials using the experimental systems available in the Laboratory of Nanomaterials Physics and Chemistry, University of Warsaw, Poland with the help of Professor Andrzej Huczko 33,34. I have also collaborated with Professor Dr. Michal Bystrzejewski from the Laboratory of Physics and Chemistry of Nanomaterials (Dept of Chemistry, University of Warsaw, Warsaw, Poland). We have improved the adsorption performance of activated carbon covalently functionalized with sulfur-containing ligands in the removal of cadmium $^{35}$. I have focused on isolation and the full-in-depth characterization of humic acids from different sources of natural origin. The humic acids were isolated from various spent materials (e.g. spent mushroom substrate). I used the following characterization techniques: scanning electron microscopy, fluorescence, and absorption UV-Vis spectroscopy, FTIR, Raman, thin layer chromatography. I have also characterized the surface chemistry by measuring the content of surface acidic groups and point of the zero charges. The manuscript is under preparation for humic acid work.

I have recently returned to Nepal after completing Fulbright Visiting Post-doctorate fellowship from Boston College, USA. During nine months, I have gathered the knowledge of Theoretical Studies on Glass-Forming Liquids: Study of a generalization of Adam-Gibbs model of relaxation in glass-forming liquids with fluctuation in the number of molecules inside the cooperative region; Systematic and comprehensive study of the configuration fraction links the excess entropy with kinetic properties described in the Adam-Gibbs model; study of the configuration fraction at the glass transition temperature in terms of the width of the distribution of relaxation times, the nonlinearity parameter, the steepness index, the excess heat capacity under constant pressure, and the number of correlated molecules or structural units. The configuration fraction in the absence of fluctuation effects has also determined for several glassforming liquids at the glass transition temperature. The Fluctuation Effects in the Adam-Gibbs Model of Cooperative Relaxation have been published recently ${ }^{36}$.

Due to crowdfunding campaign initiated by Dr. Basant Giri, Director of International Relations | Senior Scientist, Kathmandu Institute of Applied Sciences, Kathmandu, Nepal (https://fundrazr.com/efM7b?

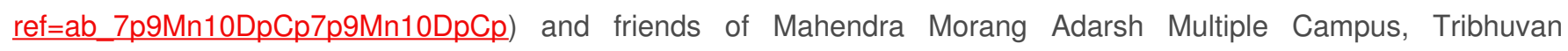
University, Biratnagar, Nepal( https://kathmandupost.com/opinion/2015/01/27/flasks-half-filled) support, we were able to purchase UV-Vis Spectrophotometer with other accessories. By using UV-Vis Spectrophotometer ${ }^{37}$, Dr. Narendra Kumar Chaudhary of our department of Chemistry started to collaborate with me and published several interesting works $38,39,40$. I not only collaborated within my own department but also with other departments within the country and outside the country $41,42,43,44$. The collaborative research work from India entitled the spectroscopic and thermodynamic interaction studies of anti-diabetic drugs with globular proteins ${ }^{45,46}$ motivated me to write thefuture research proposal on the Influence of biocompatible amino acid with mixed surfactants. 


\section{References:}

1. Ajaya Bhattarai, Parbir Nandi, Bijan Das; Journal of Polymer Research, 13, 475, 2006.

2. Ranjit De, Bijan Das, J. Chem. Eng. Data, 55, 2108, 2010.

3. Pabir Nandi, Bijan Das, J. Chem. Eng. Data, 56, 2870, 2011.

4. Parbir Nandi, Ajaya Bhattarai, Bijan Das, Journal of Polymer Sci. Part B Polymer Physics, 45, $1765,2007$.

5. Ramesh Sharma, Bijan Das, Parbir Nandi, Chanchal Das, Journal of Polymer Sci. Part B: Polymer Physics, 48, 1196, 2010.

6. A. Bhattarai, Bijan Das; Macromolecular Symposia, 315, 52, 2012.

7. A. Bhattarai, Bijan Das; Macromolecular Symposia 366, 8, 2016.

8. D.Ghosh, Ajaya Bhattarai, B. Das, Colloid Polymer Science, 287, 1005, 2009.

9. Ajaya Bhattarai, Data in Brief, 25,104365, 2019.

10. Ajaya Bhattarai, Journal of Molecular Liquids, 292, 111352, 2019.

11. Ajaya Bhattarai, Polyelectroltye-Surfactant interaction in Mixed Solvent Media, Ph.D. Thesis, 2010.

12. Sujeet Kumar Chatterjee, Lokesh Chandra Prasad, Ajaya Bhattarai; Journal of Alloys and Compounds, 496, 100, 2010.

13. Sujit Kumar Shah, Ghanashayam Srivastav, Ajaya Bhattarai, Sujeet Kumar Chatterjee; J. Nepal Chem. Soc., 24, 24, 2009.

14. Magdalena Bielawska, Aleksandra Chodzinska, Bronislaw Janczuk, Anna Zdziennicka. Colloids and Surfaces A: Physicochemical and Engineering Aspects, 424, 81, 2013.

15. Sujit K. Shah, Sujeet Kumar Chatterjee, Ajaya Bhattarai; Journal of Molecular Liquids, 222, 906, 2016.

16. Sujit K. Shah, Sujeet Kumar Chatterjee, Ajaya Bhattarai; Journal of Surfactants and Detergents 19, $201,2016$.

17. Sujit K. Shah, Sujeet Kumar Chatterjee, A. Bhattarai; Journal of Chemistry, 1, 2016.

18. Ajaya Bhattarai, Sujeet Kumar Chatterjee, Tarun Kumar Deo, Tulasi Prasad Niraula; Journal of Chemical \& Engineering Data 56, 3400, 2011.

19. Tulasi Prasad Niraula, Sujeet Kumar Chatterjee, Ajaya Bhattarai; Journal of Molecular Liquids, 250, $287,2018$.

20. Tulasi Prasad Niraula, Sujit Kumar Shah, Sujeet Kumar Chatterjee, Ajaya Bhattarai; Karbala International Journal of Modern Science, 4, 26, 2018.

21. A. Bhattarai, A. K. Yadav, S.K. Sah, Akash Deo; Journal of Molecular Liquids, 242, 831, 2017.

22. K. M. Sachin, S. Karpe, M. Singh, A. Bhattarai; Journal of Chemistry, 1, 2018.

23. K. M. Sachin, S. Karpe, M. Singh, A. Bhattarai; Macromolecular Symposia, 379, 1700034, 2018.

24. Ajaya Bhattarai, Sujeet Kumar Chatterjee, Tulasi Prasad Niraula; Springer Plus, a springer open Journal, 2, 280, 2013.

25. A. Bhattarai; Journal of Solution Chemistry 44, 2090, 2015.

26. A. Bhattarai, Kavita Pathak, Bikash Dev; Journal of Molecular Liquids, 229, 153, 2017.

27. K. M. Sachin, S. Karpe, M. Singh, A. Bhattarai; Heliyon,5, e01510, 2019.

28. K. M. Sachin, S. Karpe, M. Singh, A. Bhattarai; Royal Society Open Science, 6,181979, 2019.

29. Nitin Kumar Sharma, Man Singh, A. Bhattarai; RSC ADV, The Royal Society of Chemistry, 6, 90607, 2016.

30. A. Bhattarai, H. Wilczura-Wachnik; Journal of Surface Science and Technology 32, 17, 2016.

31. A. Bhattarai, H. W. Wachnik; International Journal of Pharmaceutics 478, 610, 2014.

32. A. Bhattarai, H. W. Wachnik; International Journal of Pharmaceutics 461, 14, 2014.

33. Andrzej Huczko, Magdalena Kurcz, Piotr Baranowski, Michał Bystrzejewski, A. Bhattarai, Sławomir Dyjak, Rita Bhatta, Balram Pokhrel, Bhim P. Kafle; Phys. Status Solidi B 250(12), 2702, 2013.

34. Andrzej Huczko, Magdalena Kurcz, Piotr Baranowski, Agnieszka Dabrowska, A. Bhattarai; J. Crystal Growth, 401, 469, 2014.

35. M. Fronczak, K. Pyrzyńska, A. Bhattarai, P. Pietrowski, M. Bystrzejewski; International Journal of Environmental Science and Technology 1, 2019.

36. Charley Hutchison, A. Bhattarai, Ailun Wang, Udayan Mohanty; Journal of Physical Chemistry B, 123(38) 8086, 2019

37. N. K. Chaudhary, P. Mishra; Journal of Chemistry,136285, 1-12, 2015.

38. N. K. Chaudhary, A. Bhattarai, B. Guragain, A. Bhattarai; Journal of Chemistry, 1-13, 2020.

39. N. K. Chaudhary, N. Chaudhary, M. Dahal, B. Guragain, S. Rai, R. Chaudhary, K. M. Sachin, R. Lamichhane- 


\section{Encyclopedia}

Khadka, A. Bhattarai; Preprints 2020, 2020050060 (doi: 10.20944/preprints202005.0060.v1).

40. N. K. Chaudhary, B. Guragain, R. Lamichhane Khadka, A. Bhattarai; Beni-Suef University Journal of Basic and Applied Sciences (in press).

41. R. K. Dev, A. Bhattarai, N. K. Chaudhary, P. Mishra; Int. J. Pharm. Sci. Rev. Res., 60(1), January - February Article No. 22, Pages: 115-121, 2020.

42. R. K. Dev, A. Bhattarai, N. K. Chaudhary, P. Mishra; Asian Journal of Chemistry (in press).

43. Indu M. Paudel, Ajaya Bhattarai; Pharmaceutical Sciences and Research, 7(1), 28-33, 2020.

44. A. Chandra, A. Bhattarai, A. K. Yadav, J. Adhikari, M. Singh, B. Giri; ChemistrySelect, 5(14), 4239-4246, 2020.

45. K. M. Sachin, S. Karpe, M. Singh, A. Bhattarai; Biointerface Research in Applied Chemistry, 9(4) 4172, 2019.

46. K. M. Sachin, S. Karpe, M. Singh, A. Bhattarai; Asian Journal of Chemistry (in press).

\section{Keywords}

Polyelectrolyte; Surfactant; Dyes 